\section{RSV-Infektion aktiviert dendritische Zellen}

\author{
Bei Kindern führt eine Infektion mit Respiratory Syncytial Virus \\ (RSV) nicht selten zu einer Bronchiolitis und einer frühen \\ allergischen Sensibilisierung. Die zellulären Auswirkungen einer \\ RSV-Infektion wurden nun bei Mäusen analysiert.
}

W eibliche Mäuse im Alter von 812 Wochen wurden nasal entweder mit RS-Viren, RSV-freien Hep2-Zellen oder durch UV-Licht inaktivierten RS-Viren beimpft. Nach 4, 6, 10, 14 und 21 Tagen wurde den Tieren das Lungen- und Milzgewebe zur weiteren Verarbeitung und Zellgewinnung entnommen.

Bei allen infizierten Tieren war 4 Tage post infectionem ein deutlicher Anstieg des RSV-Titers zu messen.
Nach 6 Tagen kam es bei den infizierten Tieren klinisch zu einer deutlichen Gewichtsabnahme, perivaskulär und peribronchiolär waren im Lungengewebe der getöteten Tiere Entzündungszeichen sichtbar. Bei den erst nach $21 \mathrm{Ta}-$ gen getöteten Tieren hatten sich die Entzündunsgzeichen fast vollständig zurückgebildet. Die Zahl der CD11c ${ }^{+}$Zellen stieg nach 4 Tagen deutlich an, das Maximum wurde nach 6 Tagen erreicht. Dieses Niveau wurde bis zum
14. Tag post infectionem gehalten, danach kam es bis zum 22. Tag zu einem Abfall. Gegenüber den nicht infizierten Mäusen war die Anzahl der dendritischen Zellen noch immer um den Faktor 3,8 erhöht. Die detaillierten Analysen einzelner zur Immunabwehr befähigter Zellverbände zeigten ähnliche Ergebnisse.

Fazit: Im Mausmodell verbleiben nach einer RSV-Infektion reife dendritische Zellen im Lungengewebe, die in der Folge die Entstehung einer Hyperreagibilität begünstigen könnten.

Beyer $\mathbf{M}$ et al. Sustained increases in numbers of pulmonary dendritic cells after respiratory syncytial virus infection. J Allergy Clin Immunol 2004; 113: 127-33

\title{
Anti-IgE wirksam bei Asthma plus Rhinitis
}

\section{Asthma und Rhinitis sind zwei pathophysiologisch eng miteinander verbundene Erkrankungen. Die Gabe von IgE-Antikörpern bessert die Symptomatik beider Erkrankungen, weshalb jetzt Wirksamkeit und Verträglichkeit dieser Therapieform bei Patienten mit Asthma und begleitender Rhinitis untersucht wurden.}

D er erste klinisch erprobte Vertreter aus der Klasse der Anti-Immunglobulin-E-Antikörper ist Omalizumab. Omalizumab bindet an die FcERI-Domäne von freiem $\mathrm{IgE}$, wodurch die Spiegel an ungebundenem Serum-IgE um bis zu $99 \%$ sinken.

Allergologen erhoben für SOLAR (Study of Omalizumab in Concomitant Asthma and Rhinitis) die Daten von Patienten, die sowohl unter mäßigem bis schwerem Asthma mit einem Tagesbedarf von mindestens $400 \mu \mathrm{g}$ inhalativem Budesonid als auch unter mittelgradiger bis schwerer perennialer Rhinitis litten. Die rekrutierten 405 Patienten erhielten über 28 Wochen zusätzlich zur benötigten Basismedikation randomisiert entweder Omalizumab- oder PlazeboInjektionen. Die Dosierung von Omalizumab orientierte sich an Körpergewicht und individuellen IgE-Spiegeln (mindestens $0,016 \mathrm{mg} / \mathrm{kg} / \mathrm{IgE}[\mathrm{IU} / \mathrm{ml}]$ ), die Injektion erfolgte je nach zu applizierender Gesamtdosis alle 2 oder 4 Wochen.

Die zusätzlich mit dem IgE-Antikörper behandelten Patienten erlitten weniger Asthmaexazerbationen als die mit Plazebo behandelten $(20,6 \%$ vs. $30,1 \%, p=0,02)$. Auch klinisch relevan-

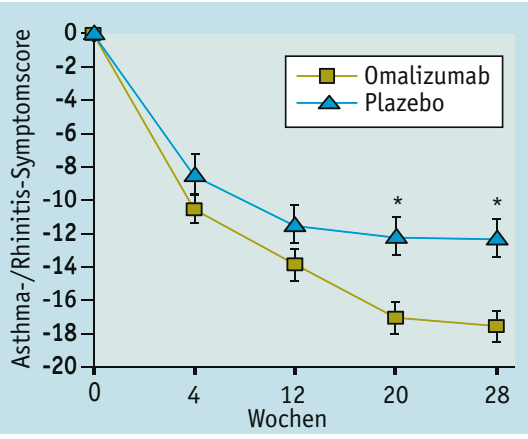

Reduktion des kombinierten Asthmaund Rhinitis-Symptomscores unter Omalizumab oder Plazebo - * $\mathrm{p}<0.001$ te Verbesserungen im Asthma Quality of Life Questionnaire und im Rhinitis Quality of Life Questionnaire waren bei den Omalizumab-Patienten häufiger als bei den Plazebo-Patienten $(57,7 \%$ vs. $40,6 \%, \mathrm{p}<0,001)$. Bei den klinischen Symptomscores (Abb.) ergab sich ebenfalls eine signifikante Überlegenheit des Verumpräparats.

Nebenwirkungen waren unter Omalizumab insgesamt häufiger als unter Plazebo, Unterschiede bei der Schwere oder den betroffenen Organsystemen bestanden aber nicht. Urtikaria und Lokalreaktionen an der Injektionsstelle traten bei vier von 209 Patienten $(1,9 \%)$ unter Omalizumab und bei einem von 196 Plazebo-Patienten (0,5\%) auf.

Fazit: Bei Patienten mit mittelschwerem bis schwerem Asthma plus perennialer Rhinitis verhindert eine zusätzlich zur Basismedikation gegebene Anti-IgEMedikation Asthmaexazerbationen und bessert die krankheitsbezogene Lebensqualität.

$b k$

Vignola AM et al. Efficacy and tolerability of anti-immunoglobulin E therapy with omalizumab in patients with concomitant allergic asthma and persistent allergic rhinitis: SOLAR. Allergy 2004; 59: 709-17 